\title{
Recent Developments of Plant Virus Research
}

\begin{abstract}
A MEETING of the Royal Society held on A March 17 was devoted to discussion of "Recent Developments of Plant Virus Research". The proceedings were opened by Dr. R. N. Salaman, who indicated the lines along which research is progressing. $\mathrm{He}$ then dealt in detail with problems arising from the existence of distinct strains within a plant virus, with special reference to potato virus $\mathrm{X}$. Six distinct strains of this virus have been isolated at Cambridge. Wide differences in virulence exist between them, but their relationship is shown by the identity of physical characters and especially by the fact that presence of any one strain in a host prevents reinfection with any other of the six strains. This protective effect implies the existence of a radical common to all $\mathrm{X}$ virus strains and distinct from any which may determine virulence. From the differences between the reactions of some strains on potato and tobacco and from the effect of mixing strains with tobacco mosaic he deduced the presence of three or four more radicals. Attention was directed to the possibility of prophylactic inoculation for protection of field crops.
\end{abstract}

Dr. K. M. Smith discussed "The Ultrafiltration of Plant Viruses". He summarized work on the determination of particle size and stressed the difficulty of interpreting data obtained by filtration of purified virus proteins through graded collodion membranes. Certain plant viruses can only be made to pass membranes of pore size exceeding $400 \mu \mu$, a result attributed to low virus concentration and loss by adsorption. Concentration of viruses leads to reduced filterability. Thus Lycopersicum virus 4 and Nicotiana virus II in fresh sap have estimated particle diameters


obtained are $80-85 \mu \mu$. With Nicotiana virus $\mathbf{I}$ the corresponding figures are 22 and $120 \mu \mu$ respectively, results partly explicable on the assumption that in purified and concentrated solutions aggregation of particles takes place. By use of a cataphoresis apparatus the last-named virus can be drawn through a membrane of $13 \mu \mu$ pore diameter. This indicates the existence of ultimate virus units having diameter about $4 \mu \mu$.

Mr. F. C. Bawden described work on purification of crystalline and liquid crystalline virus proteins. From plants infected with tobacco mosaic a nucleoprotein has been isolated, solutions of which have a satiny sheen. When such a solution is slowly concentrated, it becomes viscous, and when shaken the resultant gel breaks up into microscopic needles. A strong solution allowed to stand separates into two liquid layers; the lower and more concentrated is completely birefringent whilst the upper exhibits anisotropy of flow. The difference between the layers has thus been attributed to more perfect orientation of suspended rod-shaped particles in the lower solution. In the host cell the ' $\mathrm{X}$ ' bodies are not birefringent, but the striate material peculiar to this virus exhibits birefringence along the transverse axis and suggests formation of true virus crystals in the plant, although infected host sap represents only a $0 \cdot 2-0 \cdot 3$ per cent concentration of virus protein. Great differences may be inferred between the state of virus particles in the host and in purified pre. parations; the latter are much less infective and show greater anisotropy of flow than a clarified sap of similar concentration. The virus of tomato bushy stunt is also a nucleoprotein, but its purified prepara. tion shows no anisotropy of flow and crystallizes in perfect rhombic dodecahedra.

Prof. J. D. Bernal described the conclusions drawn from X-ray study of the structure of the virus protein molecule. No difference in intramolecular structure can be detected between liquid crystalline proteins isolated from different strains of tobacco mosaic. All have the regular internal crystalline structure characteristic of such proteins as the globulins, and their molecules apparently consist of a number of primary units of dimensions $6 \times 6 \times 6 \cdot 9 \mu \mu$. In solutions and gels the particles themselves are also regularly related to each other. The distance between them depends on the concentration of the suspension, but even in a 20 per cent solution an orderly arrangement is maintained. The minimum distance recorded, that in a dried gel, was 132 angstroms, which indicates one dimension of the particle. The corresponding figure for cucumber mosaics 3 and 4 is 135 . In plant sap, on the contrary, it was suggested that the virus units of tobacco mosaic are approximately equidimensional, with molecular weight of the order of 200,000 and a size of 120 angstroms.

Dr. MacFarlane dealt with the physical properties of vaccinia, shown by ultracentrifuging to consist of particles of unequal size. Methods were described for determining the density of vaccinia in the undried state, and it was suggested that the vaccinia particle consists of a protein molecule surrounded by an atmosphere of closely bound and orientated water ions. This atmosphere is gradually removed when vaccine is suspended in sucrose solution, while in flocculated virus no water remains. Such a structure implies the impossibility of metabolic reactions on the part of the protein nucleus.

Dr. Findlay summarized the evidence for the existence of strains in animal viruses and pointed out that such strains do not necessarily counter-immunize. Special stress was laid on variation induced by cultivation in unfavourable host tissue, but such altered strains are liable to reversion. Most apparent cases of alteration in properties are probably due to selection from a previously heterogeneous population of strains.

Mrs. Watson discussed the relation of some plant viruses to Myzus persicce. Starvation of the aphis before feeding on the infected plant results in great increase in infectivity, but this effect is lost if the duration of feeding exceeds an optimum period. In the case of Hyoscyamus virus 3 and potato virus $\mathrm{Y}$, this period is two minutes; in the case of cucumber mosaic it is fifteen minutes. No direct relationship can be demonstrated between transmissibility by $M$. persicce and concentration of virus in the plant as estimated by the starch local lesion test.

A general discussion followed, during which Prof, P. A. Murphy stressed the importance of multiplying completely virus-free potato stocks on a commercial scale. Different environments have distinctive virus populations and hence dangers arise from the use of 'masked' plant virus strains as vaccines. Dr. Elford commented on the advisability of reducing adsorption in ultrafiltration work and suggested the use of broth as a medium for re-suspension of purified plant viruses. 